\section{Leitfaden für Lüftungs- und Klimaanlagen}

D er neue überarbeitete und erweiterte "Leitfaden für Lüftungsund Klimaanlagen“ richtet sich primär an Ingenieure und Planer, technisches Personal in Vertrieb, Einkauf, Service,

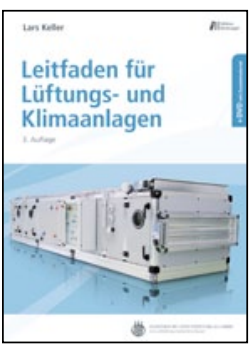
Instandhaltung, Facility Management, Studenten und Diplomanten. Im ersten Kapitel werden die thermodynamischen Prozesse aufgezeigt, das zweite Kapitel gibt eine Übersicht über die Bauteile einer Vollklimaanlage. Die Neuauflage wurde im dritten Kapitel mit nützlichen Regelschemas aus der Praxis ergänzt, die Normen und VDI-Richtlinien sind aktualisiert worden. Der aktuellen Energiesituation wurde Rechnung getragen indem die Kapitel "Regelungsmöglichkeiten bei Ventilatoren" und "Wärmerückgewinnung" grundlegend überarbeitet worden sind.

Vulkan-Verlag GmbH, Essen, www.vulkan-verlag.de

\title{
Software wertet Farben und Effekte aus
}

Eür die Analyse von Daten, die mit - dem Spektralphotometer BYKmac erfasst werden, hat die Firma BYKGardner die neue Software Smart-Lab entwickelt. Mit der Software lassen sich Farben und Effekte im Labor kontrollieren. Erfasst werden 6-Winkel-Farbe, Glitzer und Körnigkeit. Die Software ist flexibel einsetzbar und kann große Datenmengen bewältigen. Die Messungen werden im Online-Modus durchgeführt und die Ergebnisse sofort nach der Messung angezeigt. Zudem können Messergebnisse aus jeder verbundenen Datenbank geladen werden. Die Software verfügt unter anderem über eine "Standardmanagement"Funktion, mit der die Farbdifferenzmethode sowie die Toleranzen festgelegt werden. Die Ergebnisse erscheinen automatisch in einer Datentabelle und Grafik. Basierend auf ihren eigenen To- leranzen werden die Werte wie bei einer Pass/Fail-Analyse in rot, gelb und grün angezeigt. Zusätzlich können sie in einer Lab-Grafik oder einem Linienoder Farb-Travel-Diagramm dargestellt werden. Die Messdaten werden in einer SQL-Datenbank gespeichert und lassen sich mit übersichtlichen Filtern schnell nach den gewünschten Kriterien auswählen. Standards und Proben können auch in als xml-Dateien gespeichert werden.

Tel. 08171 3493-0, www.byk.com

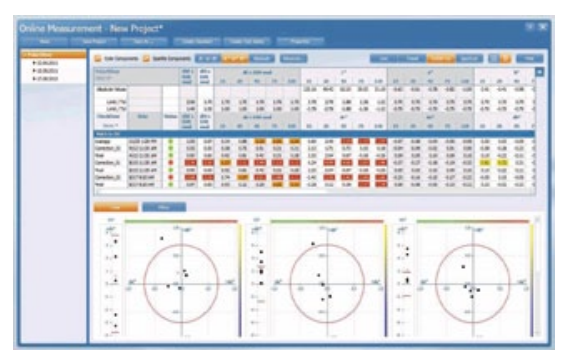

Wir sind Teil eines führenden europäischen Lackherstellers, und haben es uns zur Aufgabe gemacht mit intelligenten Lösungen, dem notwendigen Know-How und einem hohen Maß an persönlichem Engagement unsere Kunden außerordentlich zufrieden zu stellen.

Das Produktportfolio dieses Familienunternehmens lässt bei unseren Kunden praktisch keine Wünsche unerfüllt, so daß wir uns mit Fug und Recht als Systemanbieter auf dem Markt präsentieren können.

Für den Vertriebsbereich Süddeutschland suchen wir einen

\section{Technischen}

Verkaufsberater Pulverlacke $(\mathrm{m} / \mathrm{w})$ im Außendienst

\section{Was Sie erwartet:}

Sie beraten als Ansprechpartner unsere Kunden in der metallverarbeitenden Industrie. Sie sind für die Neukundegewinnung und die Betreuung der Bestandskunden verantwortlich. Dabei vertreiben Sie nicht nur unsere aktuelle Produktpalette, sondern nehmen auch Bedürfnisse und Trends des Markts wahr. Daraus erarbeiten Sie Konzepte, unsere Marktstellung zu verbessern und wirken bei zentralen Verkaufsfördermaßnahmen mit.

\section{Was wir erwarten:}

Neben einer technischen oder kaufmännischen Ausbildung besitzen Sie fundierte Kenntnisse aus dem Bereich der Industriebeschichtung. Sie verfügen über eine langjährige Vertriebserfahrung im Außendienst von Pulverlacken oder Industrielacken. Sie sind in der Lage, vertrauensvolle Kundenbeziehungen aufzubauen und setzen Ihr verkäuferisches Talent ein, um die bestehenden Umsätze entsprechend zu steigern.

\section{Wir bieten:}

Ein anspruchsvolles Aufgabengebiet mit hoher Eigenverantwortung in einem kleinen, teamorientierten Unternehmen. Die Vergütung ist über einen erfolgreichen Absatz unserer Pulverlacke jederzeit steigerungsfähig.

Ihre aussagefähige Bewerbung senden Sie bitte an

Springer-Fachmedien Wiesbaden $\mathrm{GmbH}$ Anzeigenabteilung JOT

Chiffre: 6334778

Abraham-Lincoln-Str. 46 65189 Wiesbaden 